\section{Low-profile visible intraluminal support stent-assisted embolization therapy for intracranial dissecting aneurysms: $A$ retrospective analysis of six cases}

\author{
Zhou Jia-hao,1 Seidu A. Richard,1,2 \\ Jiang Ming, 1 Deng Yin-sheng1 \\ 1Department of Neurosurgery, The \\ Affiliated Hospital of Jiangsu University, \\ Zhenjiang, P.R. China; 2Department of \\ Medicine, Princefield University, Ghana, \\ West Africa
}

\begin{abstract}
Endovascular Embolization (EVE) of aneurysms is a very effective and efficient treatment modality. Nevertheless, a few complications have been reported after EVE aneurysms has become more common. Nevertheless, its usage in treating IDAs is less reported in literature. We successfully utilized LVIS stent-assisted coils to embolize six cases of IDAs and achieved satisfactory results.
\end{abstract} of aneurysms. Our study therefore evaluated the safety and efficacy of Low-profile Visible Intraluminal Support (LVIS) stentassisted EVE for intracranial Dissecting Aneurysms (DAs). We conducted a retrospective study to identify patients with DAs who were treated with LVIS stent from July 2015 to September 2018. The DAs were categoried into ruptured and unruptured. The arteries harbouring the aneurysm were identified in all cases. LVIS device stent assisted coil EVE treatment modality was utilized to treat all the patients. Surgical safety, immediate surgery outcome, recurrence rate and imaging follow-up results of all patients were analysed. The Glasgow Outcome Scale (GOS) score of all patients where assessed during discharge. Cerebral angiography of all patients were reevaluated on scheduled visits from three months up to one year after their operations. A total of Six DA patients were identified during our analysis. Four of the cases were ruptured DAs while two cases were unruptured. The DAs originated from the Internal Carotid Artery (ICA) in two cases, while in the remaining four cases, the DAs originated from vertebral artery (VA). Stents and coils were successfully implanted in all six patients. The DAs were embolized satisfactorily and the parent arteries were patent immediately after the operations. We obsereved 5 points GOS score in four cases and 4 points in two cases. No aneurysmal recurrece, no stent collapse or displacement was obsereved in all cases during follow-ups. Our study suggests that, LVIS stent-assisted EVE is simple, safe and effective in the treatment of DAs.

\section{Introduction}

There are many intravascular treatment modalities for intracranial Dissecting Aneurysms (IDAs). ${ }^{1}$ These includes single stents, multiple stents, dense mesh stents, blood flow diverting devices, stent grafts, and coils or balloon-bearing arterial occlusions. ${ }^{1}$ In recent years, a new type of intracranial stent, Low-profile Visible Intraluminal Support (LVIS) stent, a selfexpanding nickel-titanium alloy stent, was introduced.2-4 The function of LVIS stent is based on the traditional laser stent design and the dense mesh stent technology.5 Its platinum coverage is higher than that of the traditional stent and it has a peculiar blood flow diversion. 2,5 LVIS stent-assisted endovascular embolization (EVE) of

\section{Materials and Methods}

A retrospective study was conducted from July 2015 to September 2018 to explore the safety and effectiveness of LVIS (Microvention, Tustin, California, USA) stent-assisted coil EVE on dissecting aneurysms (DAs). The ethical committee of the affiliated Hospital of Jiangsu University approved our study. All the patients and their relatives were dually informed about our intention to involve them in a study and they fully concerted to the use of their documents. A written informed consent was signed by all the patients.

Patients with DAs were included in our analysis. The DAs were categoried into ruptured and unruptured. Their demographic data as well as the arteries harbouring the aneurysm were documented. Preoperative Subarachnoid Hemorrhage (SAH) was confirmed by Computer Tomography (CT) while cerebral infarction was confirmed by Magnetic Resonance Imaging (MRI). Digital subtraction angiography (DSA) was used to confirm the location of the DAs. LVIS device stent assisted coil EVE treatment modality was utilized to treat all the patients. Surgical safety, immediate surgery outcome, recurrence rate and imaging follow-up results of all patients were analysed.

\section{Antiplatelet Medication Regimen}

DAs patients with hematomas were given $100 \mathrm{mg}$ of oral aspirin and $75 \mathrm{mg}$ of clopidogrel for at least 5 days before the
Correspondence: Ming Jiang, Department of Neurosurgery, The Affiliated Hospital of Jiangsu University, ZhenJiang, Jiefand Road, Jiangsu Province, P.R. China.

Tel: +8613775556265

E-mail: jjmm750727@163.com

Key words: IDAs; LVIS stent; endovascular, interventional, therapy.

Contributions: All authors contributed toward data analysis, drafting and critically revising the paper and agree to be accountable for all aspects of the work. Seidu A. Richard wrote the final paper.

Conflict of interest: The authors declare no conflict of interest.

Funding: 2017 Technology Innovation FundSocial Development Project of Zhenjiang City (SH2017017).

Ethics approval: The ethical committee of the affiliated Hospital of Jiangsu University approved our study.

Informed consent: All the patients and their relatives were dually informed about our intention to involve them in a study and they fully concerted to the use of their documents. A written informed consent was signed by all the patients.

Received for publication: 25 October 2019.

Revision received: 5 February 2020

Accepted for publication: 30 June 2020

This work is licensed under a Creative Commons Attribution NonCommercial 4.0 License (CC BY-NC 4.0).

(C) Copyright: the Author(s), 2019

Licensee PAGEPress, Italy

Neurology International 2020; 12:8346

doi:10.4081/ni.2020.8346

surgery. All the patients were nursed at the neuro-intensive care unit before surgery. Patients with unruptured aneurysms were put on $300 \mathrm{mg}$ of aspirin and $300 \mathrm{mg}$ of clopidogrel a day before the surgery.

\section{Surgical procedure}

All the operations were performed under general anesthesia. The patients were heparinized (un-fractioned heparin sodium, $50-70 \mathrm{u} / \mathrm{kg}$ ) before the introduction of a $6 \mathrm{~F}$ arterial sheath into the femoral artery. The $6 \mathrm{~F}$ guiding catheters were always preferentially selected to enter the necks under the guidance of the super-sliding guidewires. The stent catheters were preferentially selected to march the micro-guidewires with the aim that, the stents crossed the DA segments. Also, the appropriate diameters 
and lengths of the stents were selected according to the lengths of the diseased vessels. Furthermore, the angles of the dissecting aneurysmal necks and the diameters of the arteries involved were also taken into consideration. In all cases, the microcatheters with the coils were guided into the aneurysmal lumens with the aid of microguidewires, and the stents released in to the DAs.

\section{Postoperative follow-up}

We evaluated the degree of embolization of the DA immediately after surgery according to the Raymond grading standard. ${ }^{6}$ Complete embolization was grade 1 , subtotal embolization was grade 2 while partial embolization was grade 3. All patients were put on oral clopidogrel $75 \mathrm{mg}$ and oral aspirin $100 \mathrm{mg}$ for at least 3 months. The Glasgow Outcome Scale (GOS) score of all patients where assessed during discharge. Cerebral angiography of all patients were reevaluated on scheduled visits from three months up to one year after their operations.

\section{Results}

\section{Baseline characteristics}

A total of six DA patients were identified within 3-years period during our analysis. The patients were five males and one female. Their ages ranged from 34-63 years with a mean age of 46.8 years. The clinical presentation in most of our patient was spontaneous. Only one patient had traumatic DA. Four of them presented with ruptured DAs and Subarachnoid Hemorrhage $(\mathrm{SAH})$ while two were unruptured. The DAs originated from the Internal Carotid Artery (ICA) in two cases, while in the remaining four cases the DAs originated from Vertebral Artery (VA). In three cases, the ruptured DAs were found on the VA and their occurrences were spontaneous. Also, in one case, the ruptured DA was found on the ICA and the occurrence was traumatic in nature. The remaining two cases were unruptured DAs. One of unruptured DAs was in the ICA while the other was in the VA. One of the aneurysms in VA was found incidental during physical examination and cerebral infarction was confirmed on radiology in the same patient (Table 1).

DSA revealed that, the origin of hematomas in four patients with DAs were the posterior circulation. Out of the four, the hematomas were located far from Posterior

\section{Outcomes of stent assisted emboliza- tion procedure}

In all cases, the operations were successfully completed. Six LVIS stents were

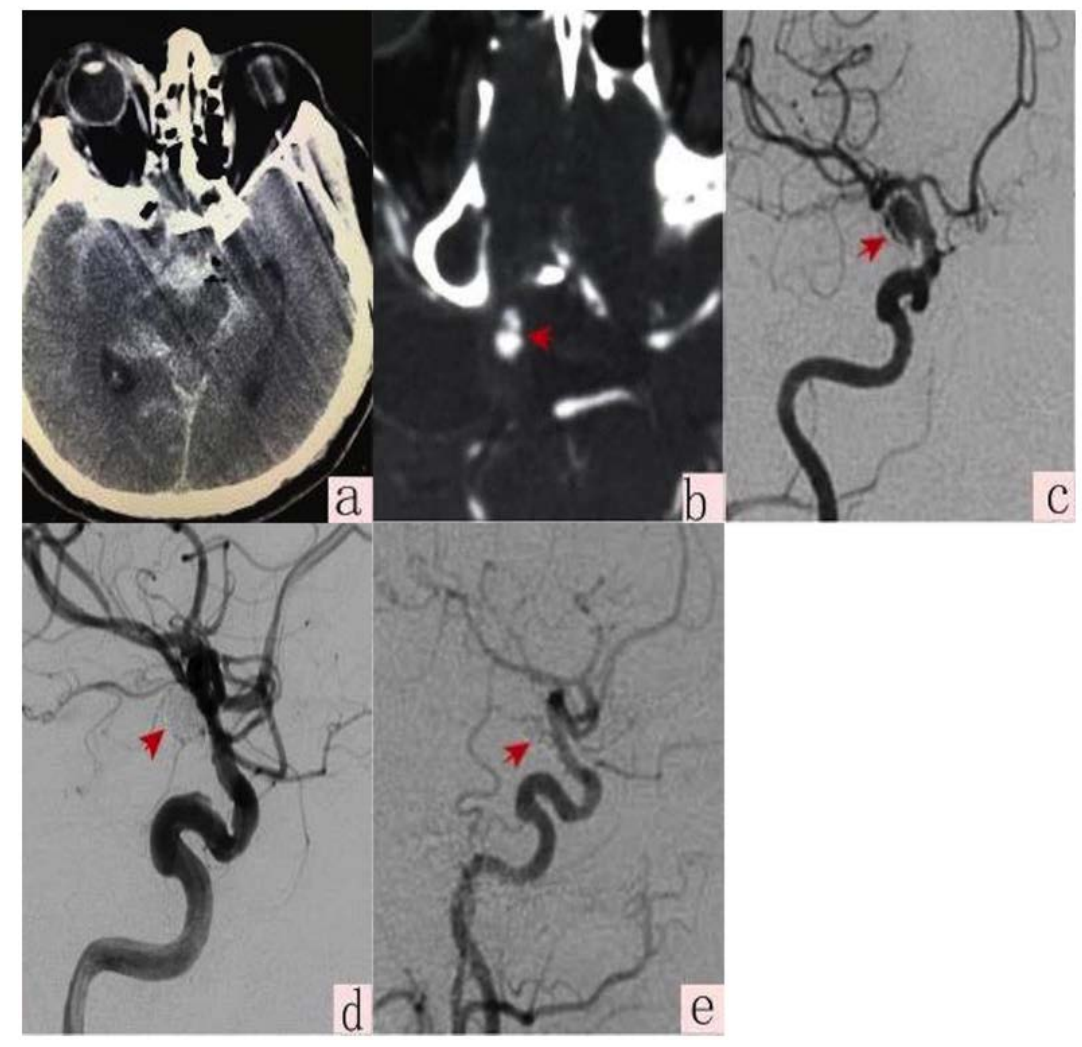

Figure 1. Right internal carotid artery end-dissection aneurysm treated with LVIS stentassisted coil embolization: a. Head CT scan showing a right-sided cavernous sinus hemorrhage or subarachnoid hemorrhage; b. Cranial CTA showing the double cavity sign; $c$. Cerebral angiography showing the right internal carotid artery end bulging aneurysmal expansion, with far end vessel stenosis; d. Shows LVIS stent assisted coil aneurysm embolization and immediate cerebral angiography after stenting angioplasty; e. The dissection aneurysm did not recur four months after cerebral angiography and the diseasebearing vessels are not narrowed either. Note: red arrows show coils in the aneurysmal sac.

Table 1. General information of patients.

\begin{tabular}{llll} 
Patients & Onset & Imaging Characteristics & Location of aneurysm dissection \\
1 & Spontaneous & DA + Hematoma & Far from the PICA \\
2 & Spontaneous & DA + Infarction at the Medulla oblongata & Far from the PICA \\
\hline 3 & Spontaneous & DA + Hematoma & Far from the PICA \\
4 & Spontaneous & DA + Hematoma & Around the PICA \\
\hline 5 & Detected on PE & DA & Cl-C2 segment of Internal carotid artery \\
\hline
\end{tabular}

DA=Dissecting aneurysm, PICA=Posterior inferior cerebellar artery, LVIS=Low-profile visible intraluminal support. PE=Physical examination. Preoperative subarachnoid hemorrhage (SAH) was confirmed by computer tomography (CT) while cerebral infarction was confirmed by magnetic resonance imaging (MRI). Digital subtraction angiography (DSA) was used to confirm the location of the DAs. 
implanted in the diseased vessels. All the stents were smoothly delivered. The stents passed through the curved vessels smoothly, and had good self-expanding and supporting forces. The stents were well adhered to arteries and the coils filled the aneurysmal sacs. There were no deformation or displacement of the stents after the coiling (Figure 1). We observed $66.7 \%(n=4)$ in grade 1 and $33.3 \%(n=2)$ in grade 2 in accordance to the Raymond classification. This classification modality assessed the degree of embolization of the DA immediately after surgery. No fresh hemorrhages or cerebral infarctions were observed during and immediately after the operations in all cases. We also did not observe any perforations or neurological deficit throughout the postoperative period as well as follow-up visits. We observed modified GOS score of 5 points in four cases and a score 4 points in two cases.

\section{Outcomes of follow-up visits}

No mortality was recorded throughout our follow-up visits and all patients are still alive and well. Also, no rebleeding or cerebral infarction was observed in any of our patients. In all patients, neurological deficits improved with no further deficits. Follow-up imaging showed no recurrence of DAs and in all patients, the diseased vessels were patent with no stenosis at ends of the stents (Figure 2).

\section{Discussion}

Arterial dissection refers to the infiltration of blood into the wall of the blood vessel via damaged intima of the artery resulting in intramural hematoma. ${ }^{7}$ When the intima and media are involved, the vascular lumen may be narrowed or even occluded. 7,8 Blood infiltration into the adventitia often results in bulging of the wall leading to the formation of a sandwich aneurysm. ${ }^{7}$ Furthermore, the outer layer of the bulging arterial wall is often weak with no tissue support around it making it prone to dissection. 7,8 IDAs are special types of dissection aneurysms that often results in SAH with arterial vasospasms as well as ischemic stroke. 9,10 Our study revealed that, the occurrence of DAs is very rare since we identified only six case within 3 -years period at our institution. The patients were made up of five males and one female. Their ages ranged from 34-63 years with a mean age of 46.8 years.

Wang et al., with a similar study involving 38 patients with vertebral artery dissecting aneurysms, observed neurological symptoms like syncope, headache at the occipital region, visual change, nuchal pain, vertigo, ataxia, hemiparesis as well as hemiplegia. ${ }^{11}$ The clinical presentation in most of our patient were spontaneous. Only one patient had traumatic DA. Early diagnosis and treatment of IDAs is advocated because of their high morbidity and mortality. 10,12 We did not report any mortality and all our patients recovered well after their operations with no neurological deficits. Highresolution MRI is the most modern tools for the diagnosis of IDAs. ${ }^{13}$ Nevertheless, DSA usually reveal typical images like doublechamber sign, intimal flap, bead sign as well as contrast agent retention. ${ }^{10,13}$ In our study, preoperative SAH was confirmed by

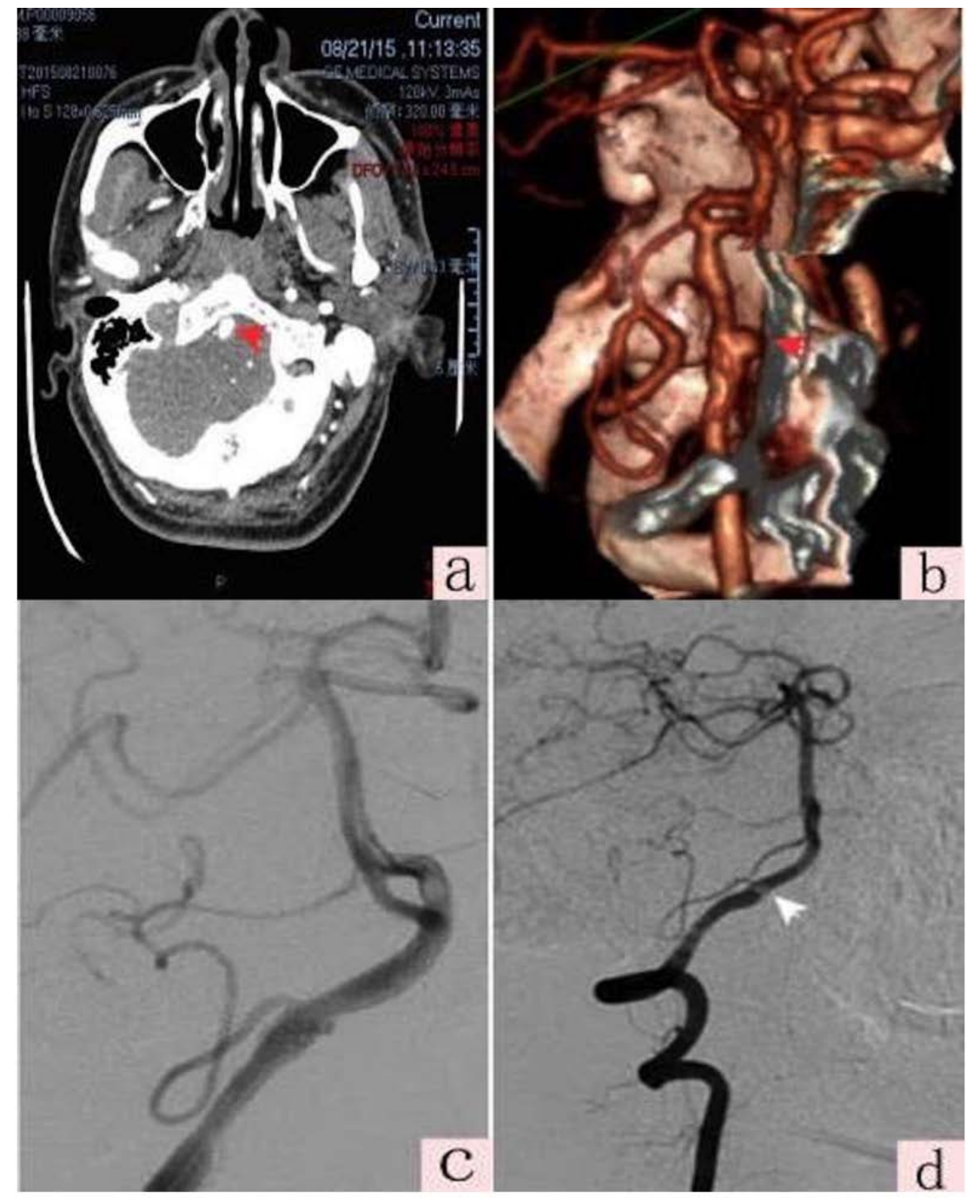

Figure 2. Right vertebral artery V4 segment dissection aneurysm treated with LVIS stentassisted coil embolization: a. Cranial CTA showing the double-chamber sign; b. Head CTA reconstruction image shows the right vertebral artery and the posterior inferior cerebellar artery with a contralateral aneurysm accompanied by distal vertebral artery stenosis; c. cerebral vascular contrast imaging 6 months after embolization; d. Cerebral angiography showing not recurrence of dissection of the aneurysm one year after the procedure. No vessel stenosis and no posterior inferior cerebellum infarction. Note: red arrows show coils in the aneurysmal sac while white arrow show patency of diseased vessel. 
flow into the aneurysmal cavity. ${ }^{12}$ Followup imaging showed no recurrence of DAs and in all patients, the diseased vessels were patent with no stenosis at ends of the stents.

Also, the potentials of causing ischemia as well as poor communication in the dominant lateral VA has been reported. 14 The repair and reconstruction of the vessel wall is achieved by reconstructing the lumen of the vessel, retaining the disease-bearing artery, avoiding obstruction of the perforated vessel, and reducing the incidence of ischemic stroke. ${ }^{15}$ We did not observe any mortality throughout our follow-up visits and all patients are still alive and well. In all patients, neurological deficits improved with no further deficits. Prior of the procedures, DAs patients with hematomas were given $100 \mathrm{mg}$ of oral aspirin and $75 \mathrm{mg}$ of clopidogrel for at least 5 days before the surgery. All the patients were nursed at the neuro-intensive care unit before surgery. On the other hand, patients with unruptured aneurysms were put on $300 \mathrm{mg}$ of aspirin and $300 \mathrm{mg}$ of clopidogrel a day before the surgery.

Endovascular treatment often involves single stent or multiple stent implantation, stent-assisted coil embolization, blood flow guiding device and stent graft implantation. ${ }^{16-18}$ In recent years, there have been many reports of endovascular treatment of IDAs. Although vascular wall reconstruction and repair have become the management trend, there are still higher risks of recurrence and bleeding after surgery. ${ }^{19,20}$ We did not observer rebleeding or cerebral infarction in any of our patients. They are many types of devices in the market for the treatment for IDAs. We preferentially used LVIS device to determine its efficiency as well as effectiveness. In our study, we achieved good results with the LVIS stents assisted coil embolization of patients with IDAs. Raymond classification modality was used to assess the degree of embolization of the dissected aneurysm immediately after surgery. We observed that $66.7 \%$ of patients had grade 1 according to the classification above while $33.3 \%$ had grade 2. Furthermore, we observed modified GOS score of 5 points in 4 cases and a score 4 points in 2 cases.

LVIS device has emerged as a new type of intracranial stent in recent years. ${ }^{21}$ These devices are flexible, braided microstent intended for the stent-assisted coiling of wide-necked intracranial aneurysms.5,22 These devices have demonstrated to be effective and efficient embolization treatment modality for intracranial aneurysms.4,21,23 The LVIS stents are selfexpanding nickel-titanium devices. The metal coverage of LVIS has been estimated to be about $23 \% .23,24$ The end of the devices has a radiopaque mark, and two coiled wires are inserted through the microcatheters. 5,24

The LVIS stents are available in a wide range of models and are suitable for almost all aneurysms. They have good compliance and vascular accesses. They also have good support for the vessel wall and mesh sizes as small as $1 \mathrm{~mm} \cdot 3,22,23,25$ The coil rings are less likely to protrude into the aneurysmal wall. Similarly, the mesh does not obstruct the rate of the blood flow through the device. This allows for hemodynamics changes and reduces the recurrence rate of the aneurysms. $3,22,24,26,27$ Nevertheless, wang et al reported post-procedural complications like pontine infarctions and delayed thromboembolic event. 11

Our study was limited because of small number of cases. Also, this was a single Centre experience with LVIS device for IDAs. Therefore, we advocate further studies on the long-term efficacy and safety of LVIS device for IDAs. Nevertheless, six DAs cases within three years means the disease is very rare.

\section{Conclusions}

In all the patients, the devices were delivered smoothly. The stents passed through the curved blood vessels smoothly, and had good self-expanding and supporting force. The stents were well attached. The coils filled the IDAs well with no deformation or displacement of the stents.

No fresh hemorrhages or cerebral infarctions were observed immediately and after the operation. Postoperative follow-up of imaging revealed no recurrences of the DA. The vessels harboring the aneurysms were patent with no stenosis. Our study suggests that LVIS devices for embolization of IDAs is simple, safe, and efficient.

\section{References}

1. Wang Y, Zhao C, Hao X, et al. Endovascular interventional therapy and classification of vertebral artery dissecting aneurysms. Exp Ther Med 2014;8:1409-15.

2. Turner RD, Turk A and Chaudry I. Lowprofile visible intraluminal support device: immediate outcome of the first three US cases. J Neurointerv Surg 2013;5:157-60.

3. Poncyljusz W, Biliński P, Safranow K, et al. The LVIS/LVIS Jr. stents in the treatment of wide-neck intracranial aneurysms: multicentre registry. J Neurointerv Surg 2015;7:524-9.

4. Shankar JJS, Quateen A, Weill A, et al. Canadian registry of LVIS Jr for treatment of intracranial aneurysms (CaRLA). J Neurointerv Surg 2017;9: 849-53.

5. Fiorella D, Arthur A, Boulos A, et al. Final results of the US humanitarian device exemption study of the low-profile visualized intraluminal support (LVIS) device. J Neurointerv Surg 2016;8:894-7.

6. Raymond J, Roy D, Bojanowski M, et al. Endovascular treatment of acutely ruptured and unruptured aneurysms of the basilar bifurcation. J Neurosurg 1997;86:211-9.

7. Ro A and Kageyama N. Pathomorphometry of ruptured intracranial vertebral arterial dissection: adventitial rupture, dilated lesion, intimal tear, and medial defect. J Neurosurg 2013; 119: 221-227.

8. Ro A, Kageyama $\mathrm{N}$ and Mukai T. Pathomorphological Examination of Patients with the Simultaneous Rupture of Dissecting Intracranial Vertebral and Intraperitoneal Arteries: Involvement of Segmental Arterial Mediolysis in Intracranial Artery Dissection. J Neuroendovascular Ther 2018;12:1618.

9. Santos-Franco JA, Zenteno M and Lee A. Dissecting aneurysms of the vertebrobasilar system. A comprehensive review on natural history and treatment options. Neurosurg Rev 2008;31:13140.

10. Mizutani T. Natural course of intracranial arterial dissections. J Neurosurg 2011;114: 1037-44.

11. Wang C-C, Fang Y-B, Zhang P, et al. Reconstructive endovascular treatment of vertebral artery dissecting aneurysms with the Low-profile Visualized Intraluminal Support (LVIS) device. PloS one 2017;12:e0180079.

12. Debette S, Compter A, Labeyrie M-A, et al. Epidemiology, pathophysiology, diagnosis, and management of intracranial artery dissection. Lancet Neurol 2015;14:640-54.

13. Neugebauer $H$ and Jüttler $E$. Hemicraniectomy for malignant middle cerebral artery infarction: current status and future directions. Int $\mathrm{J}$ Stroke 2014;9:460-7.

14. Madaelil TP, Wallace AN, Chatterjee $\mathrm{AN}$, et al. Endovascular parent vessel sacrifice in ruptured dissecting vertebral and posterior inferior cerebellar artery aneurysms: clinical outcomes and review of the literature. J Neurointerv 
Surg 2016;8:796-801.

15. Mohammadian R, Taheraghdam AA, Sharifipour E, et al. Endovascular treatment of intracranial artery dissection: clinical and angiographic follow-up. Neurol Res Int 2013; 2013:968380.

16. Hosogai M, Matsushige T, Shimonaga $\mathrm{K}$, et al. Stent-Assisted Coil Embolization for Ruptured Intracranial Dissecting Aneurysms Involving Essential Vessels. World Neurosurg 2018;119:e728-e733.

17. Li M-H, Li Y-D, Tan H-Q, et al. Treatment of distal internal carotid artery aneurysm with the Willis covered stent: a prospective pilot study. Radiol 2009;253:470-7.

18. Fang Y-B, Wen W-L, Yang P-F, et al. Long-Term Outcome of Tubridge Flow Diverter (S) in Treating Large Vertebral Artery Dissecting Aneurysms-A Pilot Study. Clin Neuroradiol 2017;27:34550.

19. Zhao K-J, Fang Y-B, Huang Q-H, et al. Reconstructive treatment of ruptured intracranial spontaneous vertebral artery dissection aneurysms: long-term results and predictors of unfavorable outcomes. PLoS One 2013;8:e67169.

20. Su W, Gou S, Ni S, et al. Management of ruptured and unruptured intracranial vertebral artery dissecting aneurysms. Journal of Clinical Neuroscience 2011;18:1639-44.

21. Zhang $\mathrm{X}$, Zhong J, Gao H, et al. Endovascular treatment of intracranial aneurysms with the LVIS device: a systematic review. J Neurointerv Surg 2017;9:553-7.

22. Zhu D, Fang Y, Yang $P$, et al. Overlapped stenting combined with coiling for blood blister-like aneurysms: comparison of low-profile visualized intraluminal support (LVIS) stent and non-LVIS stent. World Neurosurg 2017;104:729-35.

23. Cho YD, Sohn C-H, Kang H-S, et al. Coil embolization of intracranial saccular aneurysms using the Low-profile Visualized Intraluminal Support (LVIS ${ }^{\mathrm{TM}}$ ) device. Neuroradiol 2014;56:543-51.
24. Feng Z, Fang Y, Xu Y, et al. The safety and efficacy of low profile visualized intraluminal support (LVIS) stents in assisting coil embolization of intracranial saccular aneurysms: a single center experience. J Neurointerv Surg 2016;8:1192-6.

25. Behme D, Weber A, Kowoll A, et al. Low-profile Visualized Intraluminal Support device (LVIS Jr) as a novel tool in the treatment of wide-necked intracranial aneurysms: initial experience in 32 cases. J Neurointerv Surg 2015;7:281-5.

26. Möhlenbruch M, Herweh C, Behrens L, et al. The LVIS Jr. microstent to assist coil embolization of wide-neck intracranial aneurysms: clinical study to assess safety and efficacy. Neuroradiol 2014; 56: 389-395.

27. Ge H, Lv X, Yang X, et al. LVIS stent versus enterprise stent for the treatment of unruptured intracranial aneurysms. World Neurosurgery 2016;91:365-70. 